\title{
Introduction : identités, territoires et paysages ruraux en mutations
}

\section{Mark Bailoni}

\section{Q OpenEdition}

\section{Journals}

Édition électronique

URL : https://journals.openedition.org/rge/3751

DOI : $10.4000 /$ rge.3751

ISSN : 2108-6478

Éditeur

Association des géographes de l'Est

Édition imprimée

Date de publication : 31 décembre 2012

ISSN : 0035-3213

Référence électronique

Mark Bailoni, «Introduction : identités, territoires et paysages ruraux en mutations », Revue

Géographique de l'Est [En ligne], vol. 52 / 3-4 | 2012, mis en ligne le 01 juillet 2013, consulté le 21

septembre 2021. URL : http://journals.openedition.org/rge/3751 ; DOI : https://doi.org/10.4000/rge. 3751

Ce document a été généré automatiquement le 21 septembre 2021.

Tous droits réservés 


\title{
Introduction : identités, territoires et paysages ruraux en mutations
}

\author{
Mark Bailoni
}

1 La notion de territoire, espace vécu et approprié, et la perception du paysage, espace représenté et ressenti, sont des éléments fondamentaux de l'identité locale. Toutefois, les territoires et les paysages ne sont pas des ensembles figés, ils évoluent. Ces mutations, parfois brutales, peuvent être particulièrement déstabilisantes pour les populations concernées. Elles entrainent incontestablement des contrecoups sur les perceptions et les représentations identitaires. En effet, si l'identité tend à s'inscrire dans des temps longs, à travers des symboles, une culture et certaines traditions historiques, l'organisation des territoires et des paysages peut évoluer très rapidement, parfois plus vite que le renouvellement des générations.

2 Parmi ces évolutions, on peut citer :

3 - Les mutations des pratiques, des objectifs et des problématiques du monde agricole consécutives à des phénomènes multiples: modernisation et mécanisation agricole, concurrence européenne et mondiale renforcée, régulations internationales (Politique agricole commune, négociations dans le cadre de Organisation Mondiale du Commerce), multiplication des primes, scandales alimentaires et écologiques (épizooties, pollution de l'eau, etc.), question des organismes génétiquement modifiés (OGM), augmentation récente des cours, développement des biocarburants, multiplication des parcs éoliens, extension des espaces protégés et des contraintes associées pour les agriculteurs, etc.

- La restructuration des activités rurales complémentaires : raréfaction voire disparition des activités industrielles en milieu rural, réorganisation du service public et fermetures d'antennes (postes, hôpitaux, classes, etc.), disparition ou éloignement de nombreux services de proximité (commerces, médecins généralistes, etc.).

- Les évolutions démographiques : évolution, voire fin de l'exode rural dans certaines campagnes, nouveaux comportements démographiques avec l'arrivée de nouveaux habitants dans certains territoires ruraux (néoruraux, retraités, rurbains), etc. 
Les évolutions des limites villes-campagnes: phénomènes de périurbanisation, extensions des banlieues, rurbanisations, installations de grands équipements ou d'activités (ZI, ZAC) en zone périurbaine, phénomènes de métropolisation et d'archipélisation urbaine.

- Les évolutions des relations villes-campagnes : nouvelles perceptions de la ruralité en milieu urbain (tourisme vert, besoin d'« authenticité » et d'espaces de loisirs) et de la ville en milieu rural (développement de nouvelles peurs, etc.).

- Les réformes de la géographie administrative et politique : décentralisation, interrogations sur la pertinence de certains territoires politiques peu peuplés (cantons, communes), généralisation des communautés de communes, apparition des pays, mise en place de programmes de développement des territoires.

9 L'origine, la nature et l'intensité de ces mutations dépendent fortement des caractéristiques géographiques des espaces ruraux - par exemple leur situation par rapport aux grands pôles urbains ou leur accessibilité - et du contexte économique et politique - avec des différences significatives entre l'Europe du Nord-Ouest, les régions méditerranéennes et les Etats post-communistes d'Europe centrale et orientale (voir l'article de Nicoleta Ticana dans ce numéro).

10 Ces évolutions à la fois économiques, fonctionnelles, démographiques, politiques et sociales ont des conséquences majeures sur l'organisation et le dynamisme des espaces ruraux. Les mutations agricoles, et même la désindustrialisation (voir l'article de Simon Edelblutte et de Johann Legrand dans ce numéro), ont par exemple accéléré le déclin de certains territoires, alors que d'autres apparaissent très attractifs et particulièrement convoités, surtout s'ils se situent à la périphérie des villes. Ces évolutions entrainent ainsi de nouveaux enjeux et de nouvelles problématiques d'aménagement du territoire. Comment freiner le déclin de certains espaces ruraux reculés? Comment répondre aux défis de la périurbanisation?

11 Ces mutations ont parfois été initiées par les populations locales, mais elles découlent aussi de facteurs, de contextes et de décisions extérieurs à ces territoires, exacerbant les ressentiments. Mais qu'elles leur soient imposées ou non, ces évolutions ont incontestablement eu des conséquences sur les perceptions, les comportements et les représentations identitaires des populations concernées. Alors que certains phénomènes (pratiques agricoles, rurbanisation et constructions de lotissements, etc.) tendent à uniformiser les paysages, la préservation, la célébration, voire la réinvention, du patrimoine, de la culture et des traditions locales deviennent des enjeux forts et de véritables projets de territoire, même si cela peut parfois s'apparenter à une forme de repli identitaire (voir l'article de Sylvain Estager dans ce numéro). De nouvelles rivalités de pouvoirs et des réactions de rejet peuvent également apparaitre, pour contester l'arrivée de nouvelles populations, le développement de projets d'aménagement, les phénomènes de pression foncière dans certains espaces convoités, ou tout ce qui est perçu comme nuisible ou dégradant l'environnement et les paysages. Les conflits d'aménagement et les réactions de type "nimby" sont en effet très nombreux dans les espaces ruraux en mutation, particulièrement dans les territoires périurbains (voir l'article de Mark Bailoni dans ce numéro).

12 Au delà d'une étude des mutations stricto sensu des espaces et paysages ruraux en Europe, déjà accomplie dans plusieurs ouvrages, colloques et revues, ce numéro de la 
RGE étudie leurs conséquences et les enjeux qu'elles entrainent, en termes de renouveau des territoires, de perceptions et de représentations identitaires.

Si les grandes restructurations agricoles apparaissent comme les principales causes des mutations économiques des espaces ruraux, les bouleversements dans les secteurs tertiaires et surtout industriels y ont également contribué. Dans leur article, Simon Edelblutte et Johann Legrand évoquent ainsi la proto-industrie et l'industrie en milieu rural, notamment dans les espaces reculés, à travers la question de la patrimonialisation de cet héritage - en France, les campagnes ont été, comme les espaces urbains, frappées par la désindustrialisation. Si les activités industrielles sont restées marginales en milieu rural et souvent très ponctuelles, un long oubli et une faible pression économique et foncière sur les espaces ruraux ont permis de préserver un " patrimoine industriel vernaculaire " relativement important et très diversifié. Se pose alors la question du devenir de cet héritage. Les auteurs montrent que la préservation et la mise en valeur de ces éléments patrimoniaux dépendent de la sensibilisation locale, du contexte économique des territoires, des initiatives privées, de l'implication des pouvoirs publics et des potentiels touristiques. La restauration, si elle existe, répond souvent à des projets de faible envergure.

Simon Edelblutte et Johann Legrand montrent qu'une véritable culture industrielle et ouvrière peut aussi perdurer dans certains espaces ruraux. Les activités traditionnelles sont en effet des éléments fondamentaux de l'image des territoires, des identités et des cultures locales. La célébration des spécificités et des traditions économiques locales devient alors instrument de promotion du territoire. Simon Edelblutte et Johann Legrand le montrent à travers l'exemple de l'industrie dans les Boutières, en Ardèche, et Sylvain Estager à travers l'initiative locale très originale de réimplantation de la vigne à Champlitte, petite commune de Haute-Saône. Dans ces deux cas, maintenir les spécificités culturelles locales apparait essentiel pour l'image du territoire, pour préserver un dynamisme économique et mobiliser ainsi tout le tissu local.

À Champlitte, si des parcelles sont replantées et le vin commercialisé, la vigne est également célébrée au cours des fêtes viticoles et religieuses de la Saint Vincent. Cet évènement fédérateur entend perpétuer les traditions locales, mais permet également de façonner l'image de la commune, devenue le «haut-lieu » de la viticulture en HauteSaône. La réimplantation de la vigne, projet de territoire, permet une « renaissance » qui se veut à la fois économique et véritablement identitaire. Mais Sylvain Estager montre que cette renaissance se construit en partie sur des artifices. «La référence mémorielle ne serait qu'un alibi, une survivance revisitée qui rend compte non pas d'une tradition qui perdure mais d'un présent qui revisite le passé, le redéfinit, puis se le réapproprie ». D'ailleurs, en dehors de Champlitte, les perceptions sont parfois négatives : la qualité du vignoble est contestée et les fêtes viticoles sont qualifiées de réactionnaires. "Champlitte et sa célébration de la vigne témoignent au final d'un processus de production d'idéologies territoriales et des enjeux qui en découlent ».

Pour aborder la question des mutations des espaces ruraux, il apparait incontournable d'évoquer les effets de la périurbanisation. Ce processus bouleverse les territoires et les paysages, mais également les mentalités et les perceptions, parfois de manière radicale et brutale. Dans son article, Nicoleta Ticana analyse la périurbanisation autour de Bucarest. Elle décrit les grandes évolutions des franges urbaines des villes roumaines, après la chute du régime communiste, la fin des politiques d'aménagement strictes imposées par l'Etat (la planification, la «systématisation urbaine et rurale», etc.), la 
libéralisation de l'économie et le développement d'un marché foncier. La périurbanisation s'est ainsi accélérée depuis le début des années 1990, accompagnant une évolution des formes du bâti et des modèles de logement. Ce processus bouleverse les équilibres démographiques entre Bucarest et sa périphérie, mais également les dynamiques économiques, avec l'implantation de nouveaux pôles d'emploi aux portes de la ville, sur d'anciens espaces agricoles.

Nicoleta Ticana montre aussi qu'une périurbanisation trop rapide et mal contrôlée a provoqué de nouveaux problèmes d'aménagement en Roumanie, comme le dysfonctionnement des réseaux et l'insuffisance des infrastructures en périphérie des villes, ou la difficulté de maîtriser l'étalement urbain. L'auteure souligne d'ailleurs le manque de coopération, ou l'absence de structures de gouvernance adéquates pour aménager de manière concertée et efficace les franges urbaines. La spéculation et le contexte économique plus général ont également entrainé un phénomène nouveau, la crise immobilière et foncière, qui complique encore la gestion des espaces périurbains. Finalement, malgré l'expérience des villes d'Europe de l'Ouest, la périurbanisation dans les anciens pays communistes semble provoquer les mêmes problèmes et entrainer les mêmes enjeux.

En effet, des problématiques tout à fait similaires existent autour de l'agglomération londonienne, et sont au cœur des stratégies d'aménagement du territoire depuis l'entre-deux-guerres au Royaume-Uni. Dans son article, Mark Bailoni revient sur ces enjeux et sur les conséquences réellement géopolitiques des évolutions fonctionnelles, démographiques et paysagères des périphéries rurales de Londres. En effet, alors que la capitale britannique rayonne sur une très vaste région métropolitaine, aménager sa frange périurbaine est devenu une question très sensible et très conflictuelle. À travers les exemples de plusieurs projets, comme l'extension de l'aéroport d'Heathrow ou la construction d'une ligne à grande vitesse entre Londres et Birmingham, il montre qu'il est devenu quasiment impossible d'aménager ce territoire, tant la contestation est systématique et mobilisatrice. Dans cet espace périurbain très prospère, la question de l'aménagement se confronte à de multiples enjeux économiques parfois contradictoires, à des calculs électoraux et politiques, à des préoccupations environnementales, au rejet des riverains et à la défense des paysages - souvent rêvés d'une campagne anglaise, considérée comme un véritable symbole identitaire national. En cela, il reflète assez bien la situation dans de nombreux territoires ruraux européens, où les grands projets de construction d'infrastructures (ligne TGV LyonTurin, aéroport de Nantes à Notre-Dame-des-Landes, etc.) se heurtent presque systématiquement à une opposition résolue.

démarches méthodologiques très différentes, les quatre articles de ce numéro développent ainsi des exemples très différents, à partir de territoires situés dans des espaces ruraux profonds ou dans un milieu périurbains, en France, mais aussi dans un État post-communiste d'Europe orientale et au Royaume-Uni, peu agricole, mais qui conserve un attachement profond à sa campagne. Ces différents cas d'études et ces différentes approches permettent d'appréhender la diversité des enjeux spatiaux, sociaux, économiques et même géopolitiques liés à la mutation des espaces ruraux en Europe.

20 Enfin, ce numéro de la RGE se conclue par un article varia de Tobias Chilla et de Christian Schultz sur les réformes fondamentales du système d'aménagement du territoire au Luxembourg. Petit Etat à très forte dynamique économique et 
démographique, le Luxembourg constitue un véritable laboratoire d'étude pour la gestion de l'occupation de l'espace dans un contexte de forte pression foncière et de périurbanisation rapide. Confrontés au développement rapide des zones d'habitat et des zones d'activités, ainsi qu'au problème de la gestion de la circulation des 150000 travailleurs transfrontaliers qui se rendent chaque jour au Luxembourg, les responsables de l'aménagement sont aussi en prise directe avec les mutations rapides des paysages et des territoires. Pour répondre à ces défis, le Luxembourg s'est doté depuis 1999 d'une série d'instruments nouveaux d'aménagement du territoire qui reflètent l'influence française sur le plan des institutions et la tradition allemande en ce qui concerne le contenu.

\section{AUTEUR}

\section{MARK BAILONI}

Géographe, maître de conférences - LOTERR / Université de Lorraine - mark.bailoni@univlorraine.fr 